\title{
Radiative constraints on the habitability of exoplanets Gliese 581c and Gliese 581d
}

\author{
Y. $\mathrm{Hu}^{1,2}$ and F. Ding ${ }^{1,2}$ \\ ${ }^{1}$ Laboratory for Climate and Ocean-Atmosphere Studies, Peking University, 100871 Beijing, PR China \\ e-mail: yyhu@pku.edu.cn \\ 2 Dept. of Atmospheric and Oceanic Sciences, School of Physics, Peking University, 100871 Beijing, PR China
}

Received 28 April 2010 / Accepted 18 November 2010

\begin{abstract}
Aims. The M-type star Gliese 581 is likely to have two super-Earth planets, i.e., Gl 581c and Gl 581d. The present study is to investigate their habitability constrained by radiative properties of their atmospheres and the threshold of carbon-dioxide $\left(\mathrm{CO}_{2}\right)$, assuming that the two exoplanets are terrestrial, and that they have similar outgassing processes to those of the terrestrial planets in our own solar system.

Methods. Radiative-convective atmospheric models are used. Different values of $\mathrm{CO}_{2}$ concentrations and water-vapor mixing ratios are tested.

Results. Our simulation results suggest that Gl 581d is probably a habitable planet. However, at least 6.7 bars of $\mathrm{CO}_{2}$ are required to raise its surface temperature $\left(T_{\mathrm{s}}\right)$ above the freezing point of water. In contrast, Gl 581c might have experienced runaway greenhouse, like Venus, because of its too high surface temperature and the lack of an effective cold trap for water vapor. We compare our results with other independent studies.
\end{abstract}

Key words. planetary systems - radiative transfer - planets and satellites: atmospheres - stars: individual: Gl 581

\section{Introduction}

Two super-Earth extra-solar planets were detected orbiting M-dwarf Gliese (Gl) 581 (Udry et al. 2007), called Gl 581c and Gl 581d. Since the minimum mass of G1 581c and Gl 581d is about 5.0 and $8.0 M_{\oplus}\left(M_{\oplus}\right.$ is the Earth mass), respectively, below the boundary of $10 M_{\oplus}$ between terrestrial and giant gas planets, they are considered to be two terrestrial super-Earth planets. The discovery led to great interest in establishing whether these exoplanets are habitable. von Bloh et al. (2007) and Selsis et al. (2007) estimated whether the two exoplanets are inside the habitable zone of M-dwarf Gl 581 from different points of views. The former study focused on a photosynthesis-sustaining habitable zone that is determined by the limits of photosynthetic life on the planetary surface, and the latter defined the habitable distance using radiative-convective model results. Both suggested that Gl 581c is too close to the M-dwarf and outside the habitable zone, and that Gl 581d is located at the outer edge of the habitable zone and is likely to be habitable.

The habitability of a planet can be determined by various factors, as extensively discussed in the above papers. Among them, a critical and necessary condition is the existence of permanent liquid water on the surface of a planet, which requires that the surface temperature $\left(T_{\mathrm{s}}\right)$ is above the freezing point of water $(273 \mathrm{~K})$. The temperature $T_{\mathrm{s}}$ of a planet is largely constrained by the radiative properties of atmospheric compositions, in addition to stellar radiation, the distance between the planet and its parent star, surface albedo, and so on. Major greenhouse gases, such as $\mathrm{CO}_{2}$ and $\mathrm{H}_{2} \mathrm{O}$, can greatly increase $T_{\mathrm{s}}$ by means of their greenhouse effects, as it is well known that the greenhouse effect increases $T_{\mathrm{s}}$ by about $530 \mathrm{~K}$ and $33 \mathrm{~K}$ for Venus and Earth, respectively. Assuming that the planets Gl 581c and
Gl 581d are terrestrial, and have similar processes in outgassing $\mathrm{CO}_{2}$ and $\mathrm{H}_{2} \mathrm{O}$ as those of the terrestrial planets in the solar system, the two major greenhouse gases would have important influences in determining $T_{\mathrm{s}}$ and thus the habitability of the two exoplanets.

We note that greenhouse gases for the solar system have antigreenhouse effects for M-dwarf systems because M-dwarfs have much lower effective temperatures. For example, G1 581 has an effective temperature of about $3200 \mathrm{~K}$ (Udry et al. 2007), much lower than the Sun's value of $5800 \mathrm{~K}$. According to Wien's law, the radiation spectrum of Gl 581 peaks at about $0.9 \mu \mathrm{m}$ in wavelength, which is in the near-infrared (NIR) region. This redshift of the radiation spectrum causes absorption of incident stellar radiation by $\mathrm{CO}_{2}$ and $\mathrm{H}_{2} \mathrm{O}$ in the upper atmospheres of planets orbiting M-dwarfs because both $\mathrm{CO}_{2}$ and $\mathrm{H}_{2} \mathrm{O}$ have strong absorption bands in the NIR region (Goody \& Yung 1989; Yung \& DeMore 1999). Thus, for M-dwarf stellar radiation, both $\mathrm{CO}_{2}$ and $\mathrm{H}_{2} \mathrm{O}$ have not only greenhouse effects but also antigreenhouse effects. It is similar to the anti-greenhouse effect of organic aerosols in the atmosphere of Titan (McKay et al. 1991). The latter warms the upper atmosphere, but cools the surface of planets orbiting M-dwarfs. This is unlike the situation in the solar system. Solar radiation has a peak wavelength of about $0.55 \mu \mathrm{m}$ in the visible region. Both $\mathrm{CO}_{2}$ and $\mathrm{H}_{2} \mathrm{O}$ are nearly transparent to solar radiation and trap only infrared radiation from planets, which warms the planet's surface. Therefore, to assess how surface temperatures of Gl 581c and Gl 581d are constrained by atmospheric radiative processes we need to perform quantitative calculations with realistic radiation transfer models. Wordsworth et al. (2010) showed that less than about 10 bars of $\mathrm{CO}_{2}$ is sufficient to maintain a global mean $T_{\mathrm{s}}$ of Gl 581d above the freezing point of water with a radiative-convective model, 
Table 1. Parameters used in the present study.

\begin{tabular}{cccccccc}
\hline \hline Planets & $M_{\min } / M_{\oplus}$ & $r / r_{\oplus}$ & $g\left(\mathrm{~m} \mathrm{~s}^{-2}\right)$ & $\Gamma_{\mathrm{d}}\left(\mathrm{K} \mathrm{km}^{-1}\right)$ & $d(\mathrm{AU})$ & $S_{0}\left(\mathrm{~W} \mathrm{~m}^{-2}\right)$ & $c_{\mathrm{p}}\left(\mathrm{J} \mathrm{K}^{-1} \mathrm{~kg}^{-1}\right)$ \\
\hline G1581c & 5.06 & 1.8 & 15.3 & 15.3 & 0.073 & 955.83 & 1003 \\
G1 581d & 8.30 & 2.0 & 20.3 & 23.9 & 0.210 & 103.09 & 850 \\
\hline
\end{tabular}

Notes. $M_{\oplus}$ is Earth's mass, $r_{\oplus}$ is Earth's radius, $M_{\min }$ and $r$ are planets' minimum mass and radius, respectively. $g$ is surface gravity, $\Gamma_{\mathrm{d}}$ is the dry adiabatic lapse rate, $d$ is the mean distance between the star and planets, and $S_{0}$ is the average stellar flux.

and that the $\mathrm{CO}_{2}$ threshold is about 30 bars for conservative conditions. In another independent study, von Paris et al. (2010) obtained similar results, but with a slightly lower $\mathrm{CO}_{2}$ threshold.

In the present paper, we use a different radiative-convective model to investigate the $\mathrm{CO}_{2}$ threshold of Gl 581d to increase its $T_{\mathrm{s}}$ above the freezing point of water and examine both greenhouse and anti-greenhouse effects of $\mathrm{CO}_{2}$ and $\mathrm{H}_{2} \mathrm{O}$ in the Gliese 581 system. In addition, we also study radiative constraints on the habitability of G1 581c using another radiativeconvective model. In contrast to Gl 581d, Gl 581c is much closer to its parent star. Thus, the key concern for Gl 581c is whether its surface temperature is below the runaway greenhouse threshold $(340 \mathrm{~K})$ under conditions of a weak greenhouse effect and high planetary albedo. Models are described in Sect. 2. Simulations results are presented in Sect. 3. Discussion and conclusions are summarized in Sect. 4.

\section{Radiative-convective model}

Two radiative-convective models are used in the present study. One model is used for simulations for Gl 581d. The model was originally developed by Kasting et al. (1984a,b) for simulating dense planetary atmospheres with high levels of $\mathrm{CO}_{2}$, and later modified by Toon et al. (1989), Pavlov et al. (2000), Mischna et al. (2000), and others. The model takes into account the effects of collision-induced absorption by $\mathrm{CO}_{2}$, pressure-induced broadening of $\mathrm{CO}_{2}$ and $\mathrm{H}_{2} \mathrm{O}$ absorption, and Rayleigh scattering by $\mathrm{CO}_{2}$, and was used in radiation transfer and surface temperature simulations of the early Earth's atmosphere that likely had very high levels of $\mathrm{CO}_{2}$ (Kasting \& Ackerman 1986). It has 38 spectral intervals in the visible and near-infrared radiation and $55 \mathrm{spec}-$ tral intervals in the thermal-infrared. Infrared absorption by $\mathrm{CO}_{2}$ and water vapor is calculated from the Air Force Geophysical Laboratory tape. Hereafter, the model is denoted as VPL ${ }^{1}$.

The other model is the Santa Barbara DISORT Atmospheric Radiative Transfer (SBDART) model (Ricchiazzi et al. 1998), which is based on low-resolution band models developed for the LOWTRAN 7 atmospheric transmission code. The band models provide clear-sky atmospheric transmission from 0 to $50000 \mathrm{~cm}^{-1}$ and are derived from detailed line-by-line calculations with a resolution of $20 \mathrm{~cm}^{-1}$. Convective adjustment is added to the model using the dry adiabatic lapse rate. Since our simulations for G1 581c have atmospheric conditions similar to that of the Earth atmosphere, SBDART is a suitable model.

The radiation spectrum of Gl 581 is given by the Planck function, assuming that it is a blackbody. On the basis of updated observations (Mayor et al. 2009), we use a distance of $0.21 \mathrm{AU}$ between Gl 581d and its parent star rather than $0.25 \mathrm{AU}$ (Udry et al. 2007). The other parameters used here are all

\footnotetext{
1 Detailed descriptions of the model and relevant references can be found at

http://vpl . astro. washington . edu/sci/AntiModels/

models09.html
}

derived from observations documented in Udry et al. (2007) and Mayor et al. (2009), which are listed in Table 1. In the simulations of Gl 581d below, the volume mixing ratio of $\mathrm{CO}_{2}$ is set to $96 \%$, similar to that in the atmosphere of Venus, and the surface albedo $\left(A_{\mathrm{S}}\right)$ equals 0.15 , close to the terrestrial value, except for the specified cases. The zenith angle is set to $60^{\circ}$ following Manabe \& Strickler (1964). Thus, the calculated surface temperature approximately represents the global mean surface temperature. When determining the dry adiabatic lapse rate, $\Gamma_{\mathrm{d}}=g / c_{\mathrm{p}}$, for Gl $581 \mathrm{~d}$, the specific heat capacity $c_{\mathrm{p}}$ is taken to be $850 \mathrm{~J} \mathrm{~K}^{-1} \mathrm{~kg}^{-1}$ because we assume that its atmosphere is $\mathrm{CO}_{2}$ dominant, and its variations with altitude (pressure and temperature) are neglected (Wordsworth et al. 2010). For Gl 581c, $c_{\mathrm{p}}$ is taken as $1003 \mathrm{~J} \mathrm{~K}^{-1} \mathrm{~kg}^{-1}$ assuming that its atmospheric composition is similar to that of Earth's atmosphere. For the VPL model, 101 vertical levels in pressure coordinates are used, and for the SBDART model 50 vertical levels are used. In all simulations, models are integrated by performing iterations in which time is varied until they reach equilibrium.

\section{Simulation results}

\subsection{Simulations for $\mathrm{Gl} 581 \mathrm{~d}$}

Two types of simulations are performed for G1 581d. The first type of simulations has a dry atmosphere (no water vapor). The simulations indicate how the $T_{\mathrm{s}}$ of Gl 581d changes with increasing $\mathrm{CO}_{2}$ and how the anti-greenhouse effect of $\mathrm{CO}_{2}$ can be tested. The second type of simulations incorporates a moist atmosphere. Various levels of water-vapor mixing ratios are added to the model to test both the greenhouse and anti-greenhouse effects of water vapor on $T_{\mathrm{s}}$. We also perform simulations with fixed relative humidity $(\mathrm{RH})$, which more realistically show the dependence of water-vapor concentration on $\mathrm{CO}_{2}$ levels and their greenhouse effects on $T_{\mathrm{s}}$.

Figure 1a shows simulated vertical temperature profiles with surface air pressure $\left(P_{\mathrm{s}}\right)$ ranging from 1 to 50 bars, that is, $\mathrm{CO}_{2}$ partial pressure varies from 0.96 to 48 bars. It is found that $T_{\mathrm{s}}$ increases from $217.2 \mathrm{~K}$ to $375.6 \mathrm{~K}$ with increasing $\mathrm{CO}_{2}$. For $P_{\mathrm{s}}$ $=5$ bar, $T_{\mathrm{s}}$ is $257.8 \mathrm{~K}$. For $P_{\mathrm{s}}=10 \mathrm{bar}, T_{\mathrm{s}}$ is $290.8 \mathrm{~K}$, indicating that less than 10 bars of $\mathrm{CO}_{2}$ is sufficient to maintain $T_{\mathrm{s}}$ of Gl 581d above the freezing point of water. Linear interpolation yields a value of $P_{\mathrm{s}}=7.32$ bars for $T_{\mathrm{s}}=273 \mathrm{~K}$, which is equivalent to about 7.0 bars of $\mathrm{CO}_{2}$, when considering the $96 \% \mathrm{CO}_{2}$ mixing ratio.

One problem associated with the results in Fig. 1a is that $\mathrm{CO}_{2}$ condensation is not considered. From Fig. 1a, one can find that vertical temperature profiles fall into the solid-phase region of $\mathrm{CO}_{2}$ between about 0.2 and 7.0 bars, indicating that there would be a $\mathrm{CO}_{2}$ condensation in this layer. $\mathrm{CO}_{2}$ condensation releases latent heat, which warms the layer where condensation forms and alters the vertical thermal structures and surface temperatures. Simulations in which $\mathrm{CO}_{2}$ condensation is considered indeed contain a warmed layer between 0.2 and 7.0 bars 

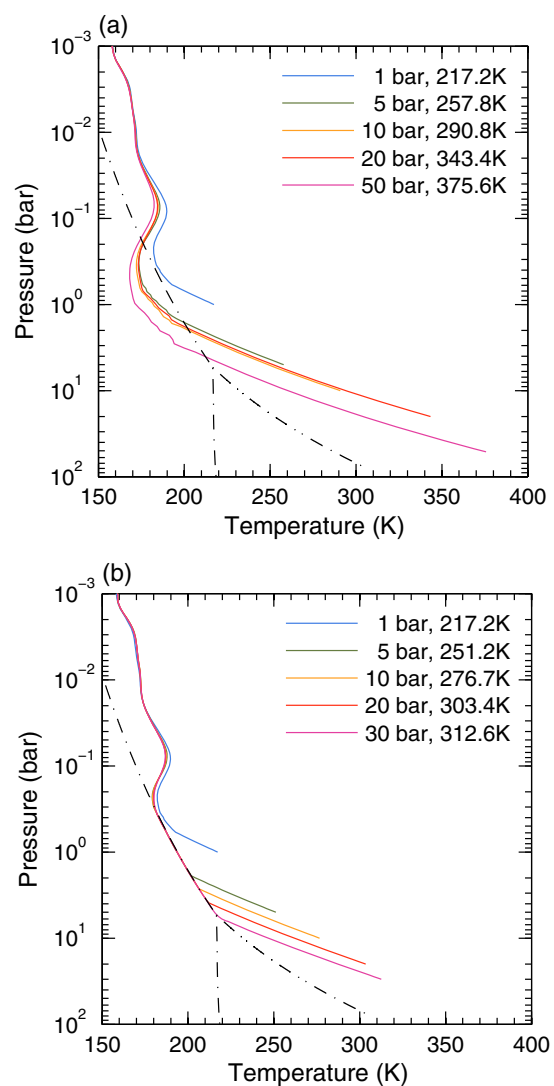

Fig. 1. Simulated vertical temperature profiles for a dry atmosphere of $\mathrm{Gl}$ 581d with different $\mathrm{CO}_{2}$ levels. Dashed-dotted lines indicate $\mathrm{CO}_{2}$ phase diagram. a) $\mathrm{CO}_{2}$ condensation is not considered, and b) $\mathrm{CO}_{2}$ condensation is considered.

and exhibit decreases in $T_{\mathrm{s}}$ (Fig. 1b). Figure $1 \mathrm{~b}$ displays lower surface temperatures than Fig. 1a. The reason why surface temperatures decrease is because the warmed layer due to $\mathrm{CO}_{2}$ condensation emits more outgoing infrared radiation at higher temperatures $\left(F=\sigma T^{4}\right.$, where $\sigma$ is the Stefan-Boltzmann constant and $T$ is air temperature), causing a weakened greenhouse effect on the surface. As a result, the $\mathrm{CO}_{2}$ threshold is higher than that without considering $\mathrm{CO}_{2}$ condensation. One can find from Fig. 1b that about 9.6 bars of $\mathrm{CO}_{2}$, i.e., $P_{\mathrm{s}}=10$ bar, is required for $T_{\mathrm{s}}$ above $273 \mathrm{~K}$.

The anti-greenhouse effect of $\mathrm{CO}_{2}$ is illustrated in Fig. 2. The black line in Fig. 2a shows the radiation spectrum of M-dwarf Gl 581 at the top of the Gl 581d atmosphere. It has a peak wavelength at about $0.9 \mu \mathrm{m}$. The red line is the incident radiation spectrum on the surface of G1 581d for $P_{\mathrm{s}}=5 \mathrm{bar}$. The red line clearly shows that a significant portion of incident stellar radiation with wavelength greater than $1 \mu \mathrm{m}$ is absorbed by $\mathrm{CO}_{2}$ before it reaches the surface. The anti-greenhouse effect of $\mathrm{CO}_{2}$ can be measured by the percentage of incident radiation absorbed by the atmosphere. The absorption percentage as a function of $P_{\mathrm{s}}$ is shown in Fig. 2b. For $P_{\mathrm{s}}=1$ bar $\left(P_{\mathrm{CO}_{2}}=0.96\right.$ bar $)$, the absorption percentage is about $20 \%$. For $P_{\mathrm{s}}=100$ bar $\left(P_{\mathrm{CO}_{2}}=\right.$ 96 bar), the absorption percentage is up to $46 \%$. Because of the anti-greenhouse effect, the upper atmosphere is warmed. The inversion layers between 0.6 and 4 bars in Fig. 1a and between 0.6 and 2 bars in Fig. $1 \mathrm{~b}$ are all due to the anti-greenhouse effect.

Rayleigh scattering of $\mathrm{CO}_{2}$ plays an important role in reducing incident radiation for atmospheres with high-level $\mathrm{CO}_{2}$, as shown by Kasting and Ackerman (1986) for the early Earth atmosphere. For M-dwarf stellar radiation, Rayleigh scattering

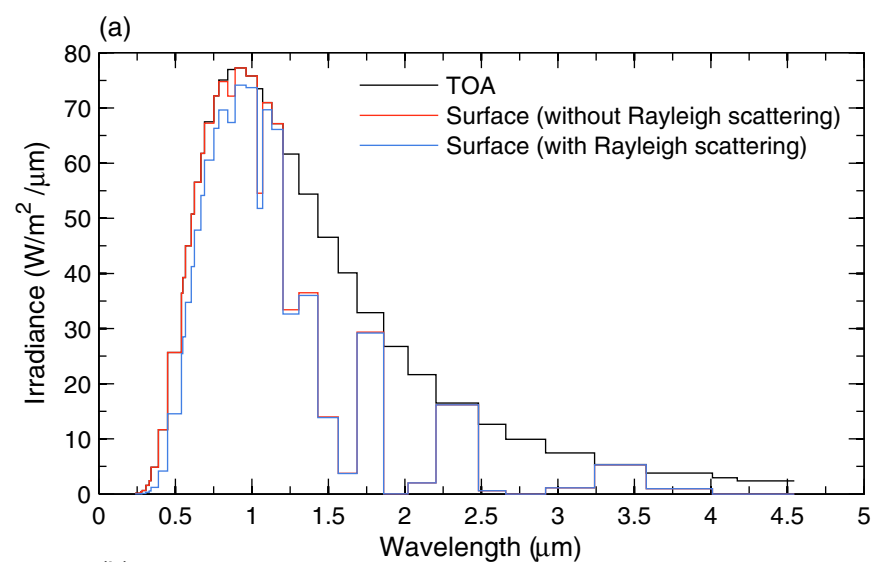

(b)

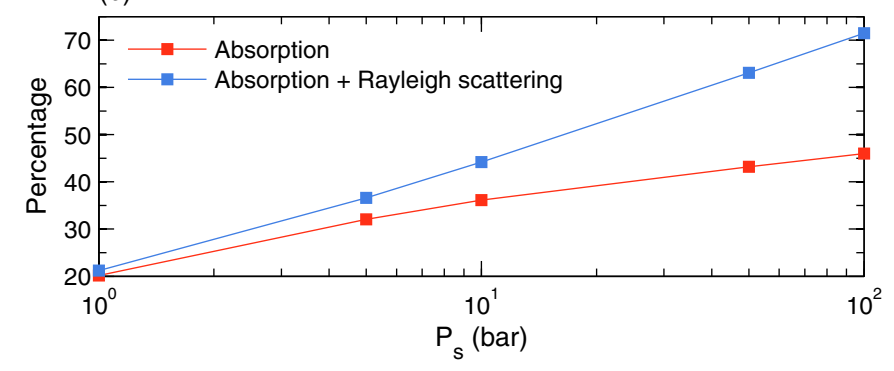

Fig. 2. a) Incident radiative spectrum of M-dwarf Gliese 581 at the top of the atmosphere (black line) and on the surface of Gl 581d for the dry condition with $P_{\mathrm{s}}=5 \mathrm{bar}\left(\mathrm{CO}_{2}\right.$ condensation is not taken into account). The red and blue lines indicate surface incident radiative spectra with and without Rayleigh scattering, respectively. b) Red line: percentage of incident radiation absorbed by $\mathrm{CO}_{2}$ as a function of $P_{\mathrm{s}}$, and blue line: percentage of incident radiation absorbed and scattered by $\mathrm{CO}_{2}$.

of $\mathrm{CO}_{2}$ is weaker because of the red shift of stellar radiation. Nonetheless, Rayleigh scattering of $\mathrm{CO}_{2}$ still has important effects in the visible region, especially for high levels of $\mathrm{CO}_{2}$. In Fig. 2a, the blue line shows the spectrum of incident radiation on the surface as $\mathrm{CO}_{2}$ absorption and Rayleigh scattering are all considered. One can differentiate between blue and red lines in the region with wavelengths shorter than $1.25 \mu \mathrm{m}$. For longer wavelengths, Rayleigh scattering quickly decays because its effect is proportional to $\lambda^{-4}$, where $\lambda$ is the wavelength of incident radiation. The blue line in Fig. $2 b$ shows the percentage of incident radiation absorbed and scattered by $\mathrm{CO}_{2}$ as a function $P_{\mathrm{s}}$. Comparison of the blue line with the red line reveals that the effect of Rayleigh scattering becomes more and more important as $\mathrm{CO}_{2}$ increases. For $P_{\mathrm{s}}=1$ bar, Rayleigh scattering has very weak effect. As $P_{\mathrm{s}}$ increases to 10 and 50 bars, it leads to a reduction in the incident radiation by $10 \%$ and $20 \%$, respectively.

The above results are for a dry atmosphere. Assuming that G1 581d has similar outgassing processes to that of terrestrial planets in the solar system, its atmosphere should also include water vapor. To examine how the greenhouse effect of water vapor, and that of $\mathrm{CO}_{2}$, influences $T_{\mathrm{s}}$, we consider $P_{\mathrm{s}}=5$ bar and fix the surface water-vapor mixing ratios to $1,10,100$, and 1000 ppmv, respectively. Water-vapor mixing ratios decay exponentially with altitudes to the same value of 1 ppmv at 0.5 bar and remain constant above this pressure level, as shown in Fig. 3b. In these simulations, convective adjustment is used with the dry adiabatic lapse rate, $\Gamma_{\mathrm{d}}=g / c_{\mathrm{p}}=23.88 \mathrm{~K} \mathrm{~km}^{-1}$. Simulation results are shown in Fig. 3a. The greenhouse effect of water vapor is strong. Even 1 ppmv of water vapor can increase $T_{\mathrm{s}}$ from $257.8 \mathrm{~K}$ for the dry atmosphere (see the green line in 


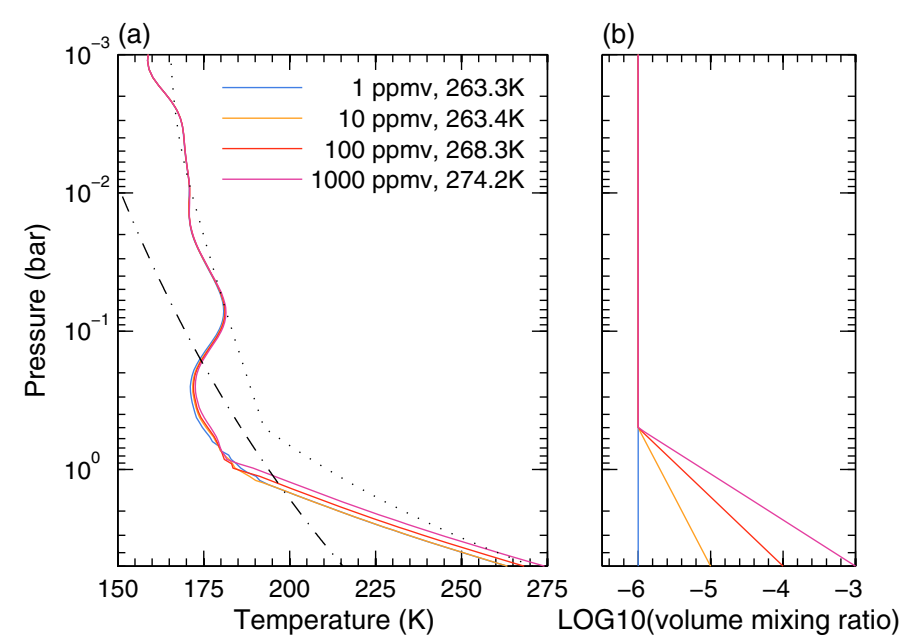

Fig. 3. a) Simulated vertical temperature profiles for a moist atmosphere of Gl 581d, with $P_{\mathrm{s}}=5$ bar and a dry adiabatic lapse rate of $23.88 \mathrm{~K} \mathrm{~km}^{-1} \cdot \mathrm{CO}_{2}$ and water vapor condensation is not considered. Dotted-line indicates the water-vapor saturation line, and the dashed and dotted-line indicates the $\mathrm{CO}_{2}$ phase-diagram between gas and solid phases. b) Vertical distributions of water-vapor mixing ratios.

Fig. 1a) to 263.3 K. For 10, 100, and 1000 ppmv of water vapor, the corresponding $T_{\mathrm{s}}$ is $263.4,268.3$, and $274.2 \mathrm{~K}$, respectively. The results suggest that a lower $\mathrm{CO}_{2}$ threshold is required as water vapor is considered.

The anti-greenhouse effect also becomes much stronger when water vapor is included. A comparison of Fig. 4a with Fig. 2a indicates that more incident radiation is absorbed by the atmosphere, especially in the region from the peak wavelength to the near-infrared region. From Fig. $4 b$, one can infer that the absorption percentage is about $32 \%$ as 1 ppmv of water vapor is added to the model, which is about $1 \%$ higher than the dry case for $P_{\mathrm{s}}=5$ bar (see Fig. 2b). For 1000 ppmv of water vapor, about $44 \%$ of the incident radiation is absorbed by the atmosphere, which represents an increase of about $13 \%$.

We note that water vapor concentration is constrained by ambient air temperatures, that is, water vapor condenses when it is saturated. Thus, one cannot arbitrarily add water vapor to the model. Figure 3 a already shows super-saturation, in which temperature profiles are below the saturation line at some levels. It suggests that the results in Fig. 3a may not be realistic. A more sophisticated way of examining how $T_{\mathrm{s}}$ is determined by water vapor, as well as $\mathrm{CO}_{2}$, is to use the method of fixed relative humidity $(\mathrm{RH})$. In this way, water vapor concentration varies with air temperatures for different $\mathrm{CO}_{2}$ levels. In these simulations below, the vertical distribution of $\mathrm{RH}$ in the troposphere is given by the formula of Manabe and Wetherald (1967) for Earth's atmosphere. This method was used to study Earth's early atmosphere by Kasting and Ackerman (1986).

Simulations with fixed RH are performed with three values of surface albedo, i.e., $A_{\mathrm{s}}=0.15,0.20$, and 0.30 . In these simulations, moist adiabatic processes are applied, and $\mathrm{CO}_{2}$ condensation is also considered. Figure 5a shows vertical temperature profiles for $A_{\mathrm{s}}=0.15$. Comparison of Fig. 5a with Fig. $1 \mathrm{~b}$ shows that the surface temperature $T_{\mathrm{s}}$ is much higher when water vapor is included in the model. For example, for $P_{\mathrm{s}}=10 \mathrm{bar}, T_{\mathrm{s}}$ is $276.7 \mathrm{~K}$ for the dry atmosphere, while it is $291.9 \mathrm{~K}$ for the moist atmosphere. This difference is due to the greenhouse effect of water vapor. However, the surface temperature for $P_{\mathrm{s}}=5$ bar, $257.9 \mathrm{~K}$, is lower than in Fig. 3 a because of the condensation of $\mathrm{CO}_{2}$ and water vapor, as well as lower lapse rates in the moist

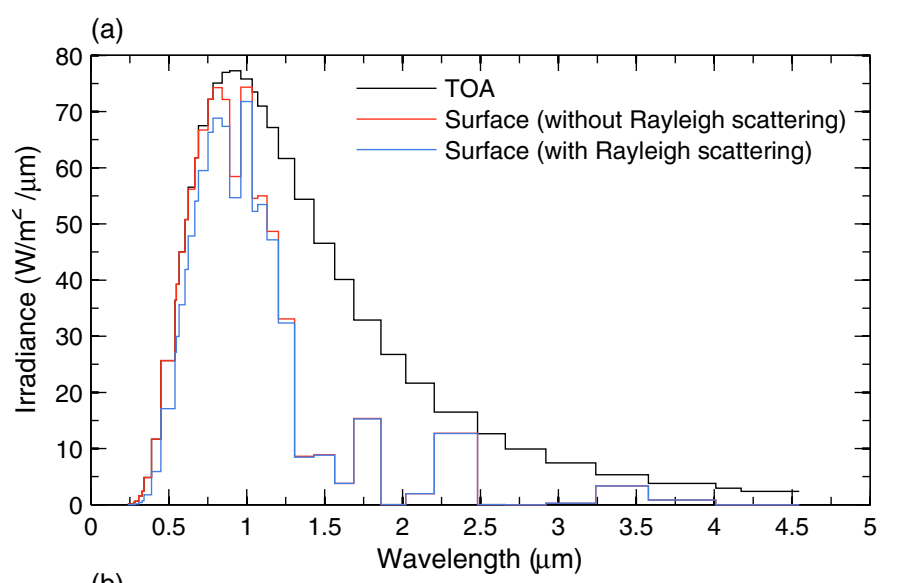

(b)

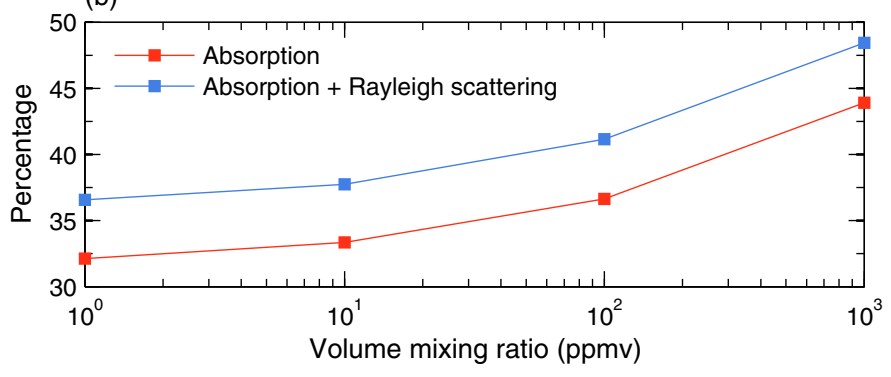

Fig. 4. a) Same as Fig. 2a, except for that 1000 ppmv of water vapor is added to the model. b) Percentage of absorption and absorption with Rayleigh scattering as a function of water vapor mixing ratio.

atmosphere. As mentioned before, the condensation of $\mathrm{CO}_{2}$ and water vapor warms the upper troposphere, causing a weakened greenhouse effect. The temperature lapse rate for moist adiabatic processes is usually lower than that in the case of dry adiabatic processes. A lower lapse rate causes a weaker greenhouse effect because outgoing infrared radiation from the upper troposphere is stronger when the air temperature is higher than for a dry adiabatic lapse rate, leading to a decrease in $T_{\mathrm{s}}$.

The temperature $T_{\mathrm{s}}$ as a function of $P_{\mathrm{s}}$, under conditions of $\mathrm{CO}_{2}$ condensation, moist adiabatic processes, and fixed $\mathrm{RH}$, is plotted in Fig. 5b. The solid-square line in Fig. 5b is for $A_{\mathrm{s}}=$ 0.15 . It crosses the dotted-line $\left(T_{\mathrm{s}}=273 \mathrm{~K}\right)$ at about $P_{\mathrm{s}}=7.0 \mathrm{bar}$, indicating that a minimum $\mathrm{CO}_{2}$ level of 6.7 bars is required for $T_{\mathrm{s}}$ to be maintained above the freezing point of water. To reach the Earth's global-mean surface temperature today (288 K), $\mathrm{CO}_{2}$ has to be about 8.64 bars $\left(P_{\mathrm{s}}=9.0\right.$ bar $)$. For $P_{\mathrm{s}}=50$ bar, $T_{\mathrm{s}}$ is $362.9 \mathrm{~K}$. One might worry that Gl 581d would undergo runaway greenhouse for $P_{\mathrm{s}}=50 \mathrm{bar}$ since the corresponding $T_{\mathrm{s}}$ is above the threshold of $340 \mathrm{~K}$ (Ingersoll 1969). However, $340 \mathrm{~K}$ is the threshold for 1 bar of surface air pressure, and the threshold increases with $P_{\mathrm{s}}$, as pointed out by Selsis et al. (2007). For surface albedos of 0.20 and $0.30, P_{\mathrm{s}}$ corresponding to $273 \mathrm{~K}$ is about 8.0 and 12.0 bars, respectively, equivalent to about 7.7 and 11.5 bars of $\mathrm{CO}_{2}$. We note that the three lines tend to converge for $P_{\mathrm{s}}=50 \mathrm{bar}$, which indicates that the effect of surface albedo on $T_{\mathrm{s}}$ becomes less important for high $\mathrm{CO}_{2}$ levels. This is because incident stellar radiation is mainly absorbed and scattered by the atmosphere $\left(\mathrm{CO}_{2}\right.$ and water vapor) for high levels of $\mathrm{CO}_{2}$, and a very minor part of incident radiation can reach the surface. Thus, $T_{\mathrm{s}}$ is mainly determined by the greenhouse effect of the atmosphere, rather than by incident radiation reaching the surface. The issue of surface albedo effect at very high levels of $\mathrm{CO}_{2}$ was also studied by Selsis et al. (2007). 

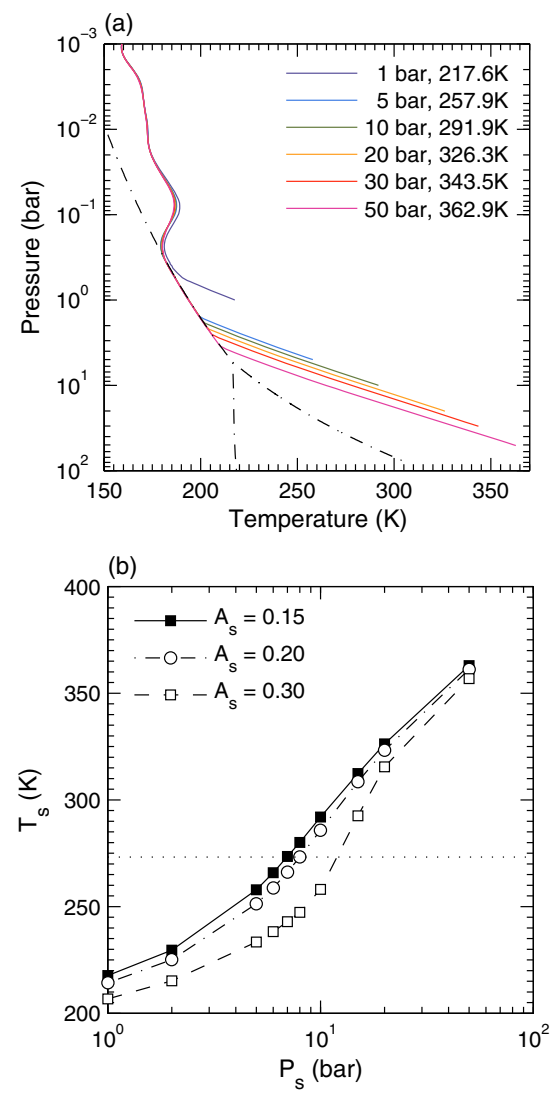

Fig. 5. a) Simulated vertical temperature profiles for a moist atmosphere for Gl 581d with fixed RH and $A_{\mathrm{s}}=0.15$. b) Simulated $T_{\mathrm{s}}$ as a function of $P_{\mathrm{s}}$ with fixed RH and moist adiabatic lapse rates.

Wordsworth et al. (2010) and von Paris et al. (2010) independently studied the $\mathrm{CO}_{2}$ threshold for Gl 581d (Hereafter, the two paper are referred as WW and vP, respectively). WW used a different radiative-convective model from ours, while vP used the same model as ours, but with different parameterizations of absorption for infrared radiation. Here, we compare these results and assess the cause of differences among these models. Figs. 6a-c compare our results and WW's for dry, moist, and water-vapor saturation atmospheres, respectively. To provide the comparison, simulations are carried out using the same parameters as that in WW and vP, such as surface albedo, temperature lapse rate, the vertical distribution of relative humidity, and so on. Figure 6a shows the comparison for the dry case (pure $\mathrm{CO}_{2}$ ). As $P_{\mathrm{s}}$ is lower than 2.5 bars, $T_{\mathrm{s}}$ in the VPL model is slightly lower than that in WW for the same level of $\mathrm{CO}_{2}$. For example, the difference in $T_{\mathrm{s}}$ is about $3 \mathrm{~K}$ for $P_{\mathrm{s}}=1.0$ bar. However, as $P_{\mathrm{s}}$ is greater than $2.5 \mathrm{bars}, T_{\mathrm{s}}$ in the VPL model is higher than in WW, and the difference in $T_{\mathrm{S}}$ between the two models increases with increasing $P_{\mathrm{s}}$. For $P_{\mathrm{s}}=20$ bars, $T_{\mathrm{s}}$ in the VPL model is about $6 \mathrm{~K}$ higher than in WW (about 292 versus $286 \mathrm{~K}$ ). For low levels of $\mathrm{CO}_{2}$, the WW model appears to have a slightly stronger greenhouse effect than the VPL model probably because Wordsworth et al. (2010) used the HITRAN 2008 database for $\mathrm{CO}_{2}$ absorption, which differs from that in the VPL model. For high levels of $\mathrm{CO}_{2}$, collision-induced absorption of $\mathrm{CO}_{2}$ becomes important and causes an enhanced greenhouse effect for $\mathrm{CO}_{2}$. The comparison indicates that the parameterization for collision-induced absorption of $\mathrm{CO}_{2}$ in WW, which is based on new measurement results (Baranov et al. 2004; Gruszka \& Borysow 1998), tends to reduce greenhouse warming for high levels of $\mathrm{CO}_{2}$. For the moist case (Fig. 6b), $T_{\mathrm{s}}$ in the VPL model is systematically lower than that in WW, and the difference also becomes greater with increasing $P_{\mathrm{s}}$. For $P_{\mathrm{s}}=$ 20 bars, the $T_{\mathrm{s}}$ in the VPL model is about $13 \mathrm{~K}$ lower than that in WW, about $332 \mathrm{~K}$ versus $345 \mathrm{~K}$. This suggests that water vapor has a stronger greenhouse effect in WW's model than in the VPL model, which increases $T_{\mathrm{s}}$ in WW's model from $6 \mathrm{~K}$ lower than in the VPL model in the dry case to $13 \mathrm{~K}$ higher than in the VPL model in the moist case for $P_{\mathrm{s}}=20$ bars. For the saturation case (Fig. 6c), $T_{\mathrm{s}}$ in WW is much higher than in the VPL model. For $P_{\mathrm{s}}=20$ bars, $T_{\mathrm{s}}$ in $\mathrm{WW}$ is about $19 \mathrm{~K}$ higher than in the VPL model, about $367 \mathrm{~K}$ versus $348 \mathrm{~K}$. This is again indicative of a stronger greenhouse effect of water vapor in WW's model than in the VPL model.

Although vP used the same model as ours, i.e., the VPL model, they changed both the parameter values and parameterizations of the model. In particular, they recomputed kcorrelated coefficients with their own line-by-line model in order to achieve a wider coverage of pressure and temperature. A comparison of our results with those of $\mathrm{vP}$ is shown in Fig. $6 \mathrm{~d}$. Our results (crosses) are also systematically lower than vP's, and the difference in $T_{\mathrm{s}}$ ranges from about $13 \mathrm{~K}$ for $P_{\mathrm{s}}=1$ bar to about $30 \mathrm{~K}$ for $P_{\mathrm{s}}=5$ or 10 bars. The new parameterization of water vapor absorption may play an important role in causing higher temperatures in vP than in the VPL model, especially for high levels of $\mathrm{CO}_{2}$. However, it does not appear to be the major reason for causing a difference in $T_{\mathrm{s}}$ for low levels of $\mathrm{CO}_{2}$, at which $T_{\mathrm{s}}$ is so low that little water vapor in the atmosphere can be present. Thus, other changes in parameters and parameterizations for $\mathrm{CO}_{2}$ are also responsible for the stronger greenhouse effect in vP. In Fig. 6d, we also determine the difference between cases of $\mathrm{CO}_{2}$ super-saturation and condensation. It is found that the $\mathrm{CO}_{2}$ super-saturation causes about a $7 \mathrm{~K}$ increase in $T_{\mathrm{s}}$ for $P_{\mathrm{s}}=20$ bars. It is difficult to directly compare the results between WW and vP because they used very different parameters. One major reason that causes higher $T_{\mathrm{s}}$ in $\mathrm{vP}$ than in $\mathrm{WW}$ is probably because surface albedo in $\mathrm{vP}$ is much lower than in WW (0.13 versus 0.2$)$. For a moist atmosphere, the models used overall by WW and $\mathrm{vP}$ have stronger greenhouse effects than the VPL model.

\subsection{Simulations for $\mathrm{GI} 581 \mathrm{C}$}

Gl 581c is much closer to its parent star than Gl 581d, at a distance of about $0.073 \mathrm{AU}$. It receives $30 \%$ more incident stellar radiation than Venus today (Udry et al. 2007; Selsis et al. 2007). Because the radiative equilibrium temperature of Gl 581c is already as high as $300 \mathrm{~K}$ for an albedo of 0.5 (Udry et al. 2007), the greenhouse effect can readily increase its $T_{\mathrm{s}}$ above the runaway greenhouse threshold, suggesting that a high planetary albedo and weak greenhouse effect are necessary for Gl 581c to be habitable. The following simulations test whether these two conditions can be realized.

First, we test the condition of weak greenhouse effect by using a low $\mathrm{CO}_{2}$ level in the model. The dash-dotted line in Fig. 7 is the simulated temperature profile for a dry atmosphere with conditions of $P_{\mathrm{s}}=1$ bar (same as that of Earth), $50 \mathrm{ppmv}$ of $\mathrm{CO}_{2}$, and $A_{\mathrm{s}}=0.5$. The corresponding $T_{\mathrm{s}}$ is $310 \mathrm{~K}$. Second, we add water vapor to the model. The vertical distribution of a watervapor mixing ratio is shown in Fig. $7 b$ (dashed-line), where the surface water-vapor mixing ratio is the same as that in Earth's atmosphere (US standard atmosphere, 1976). The reason why the water-vapor mixing ratio is set to be uniform above $700 \mathrm{hPa}$ is because the convective layer is very thin and can only reach 

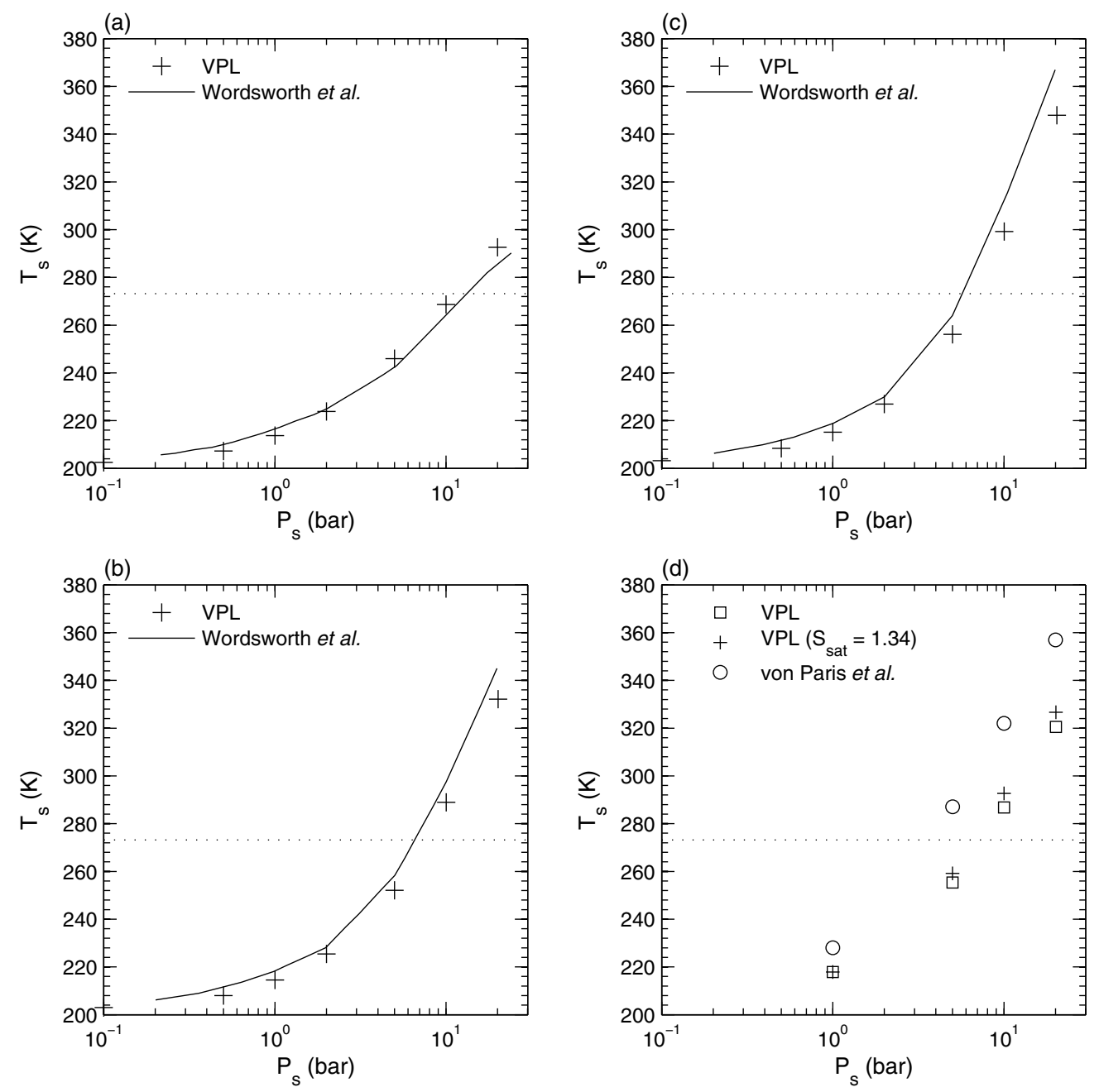

Fig. 6. Comparison of our simulation results for Gl 581d with that in WW and vP. Plots a-c compare the results of WW with our results, and plot d compares the result of $\mathrm{vP}$ with our results. a) Dry atmosphere with considering $\mathrm{CO}_{2}$ condensation, b) moist atmosphere with fixed $\mathrm{RH}$, dry adiabatic lapse rate and considering condensation of $\mathrm{CO}_{2}$, and $\left.\mathbf{c}\right)$ same as $\left.\mathbf{b}\right)$, except for saturated water vapor $(\mathrm{RH}=100 \%)$ in the troposphere. In plots a)-c), $A_{\mathrm{s}}=0.20$, gravity $g=20 \mathrm{~m} \mathrm{~s}^{-2}$. The dry atmosphere here is pure $\mathrm{CO}_{2}$, and the moist atmosphere is dominated by $\mathrm{CO}_{2}$ and contains a minor component of water vapor. The vertical distribution of RH is the Manabe-Wetherald type, with surface $\mathrm{RH}=0.77$. In plot d), parameters are same as that in $\mathrm{vP}$, that is, $A_{\mathrm{s}}=0.13$, gravity $g=23.76 \mathrm{~m} \mathrm{~s}^{-2}, \mathrm{CO}_{2}$ concentration is $95 \%$, the vertical distribution of RH is the Manabe-Wetherald type with surface $\mathrm{RH}=0.80$, and $\mathrm{CO}_{2}$ is super-saturated. Here, $S_{\text {sat }}$ indicates super-saturation of $\mathrm{CO}_{2}$. In all the plots, $T_{\mathrm{s}}$ values by WW and vP are estimated from their figures.

$700 \mathrm{hPa}$. When this amount of water vapor is added, $T_{\mathrm{s}}$ rapidly increases to $331 \mathrm{~K}$ (dashed-line in Fig. 7a). Although it remains below $340 \mathrm{~K}$, the surface RH is too low, about $3.4 \%$, to allow the existence of liquid water on the surface. To maintain permanent liquid water on Gl 581c, the surface RH must be comparable to that of Earth. However, simulations in which the fixed RH is almost identical to that used in Fig. 5 show that $T_{\mathrm{s}}$ rises quickly above $340 \mathrm{~K}$, and the model cannot reach equilibrium, which is indicative of runaway greenhouse. The above results suggest that the condition of a weak greenhouse effect can hardly be realized.

There are two possibilities of a high planetary albedo for Gl 581c: a large coverage of either snow-ice or thick clouds (Selsis et al. 2007). The former is unlikely because $\mathrm{CO}_{2}$ accumulation due to volcanic eruptions would eventually melt the snow and ice, resulting in a runaway greenhouse, even if the planet were cold initially. A high albedo due to thick clouds might be more likely because a water-rich, warm planet would have deep convective clouds (Selsis et al. 2007). However, simulations indicate that the assumption may also be unrealistic because the cloud optical thickness has to be as high as 270 to keep $T_{\mathrm{s}}$ below the threshold of runaway greenhouse.

The solid line in Fig. 7 is the simulated temperature profile, where the cloud optical thickness is 270 , the effective radius of cloud drops is $20 \mu \mathrm{m}$ (similar to terrestrial clouds), and RH is fixed as above. The simulation yields a $T_{\mathrm{s}}$ of about $338 \mathrm{~K}$, very close to the threshold of runaway greenhouse. Such a high optical thickness corresponds to a very dark world, with which there is not only little direct incident radiation but also very little scattered shortwave radiation that can reach the surface. Our calculations indeed show that only $8.76 \mathrm{~W} \mathrm{~m}^{-2}$ of scattered radiation can reach the surface (total incident radiation is about $955.83 \mathrm{~W} \mathrm{~m}^{-2}$ ), while direct incident radiation is negligible. The problem is that such thick clouds as these may not be realistic. Moreover, the above simulation is based on the assumption of $100 \%$ cloud coverage. A more realistic possibility is that strong convection must be accompanied by descending regions where there are few or no clouds. Thus, an even higher optical thickness of clouds is needed to satisfy the requirement of a high planetary albedo. 
(a)

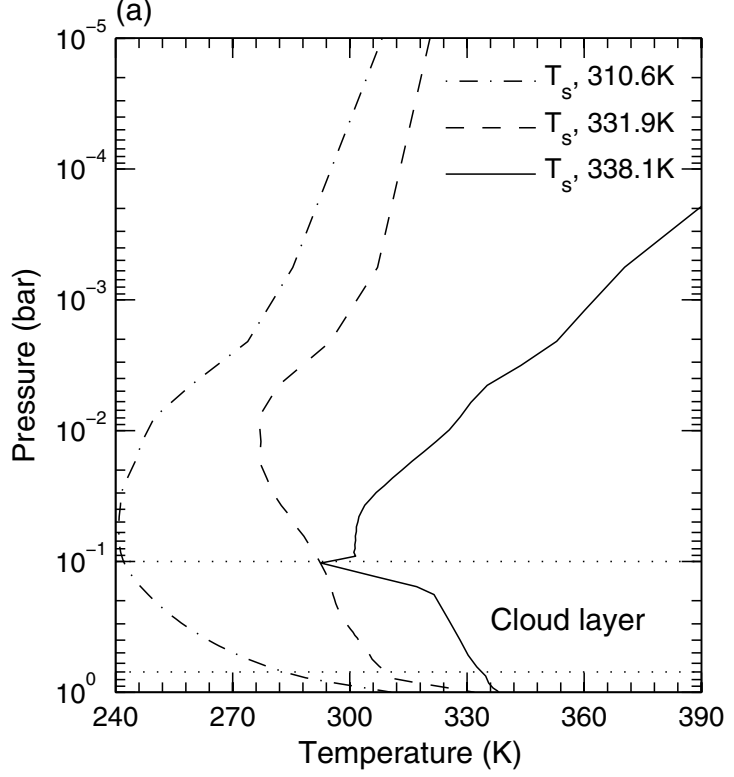

(b)

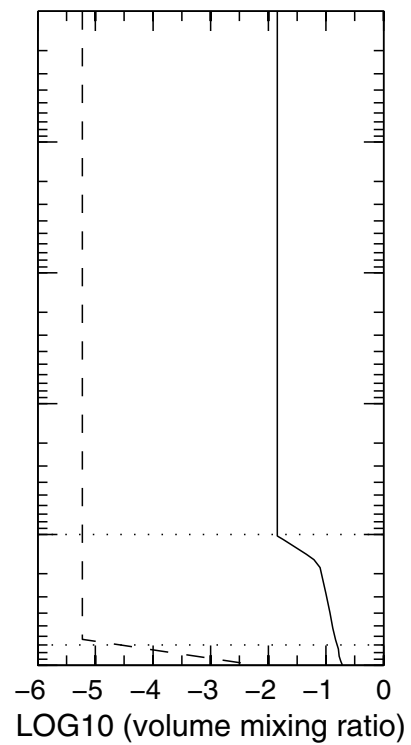

Fig. 7. a) Simulated vertical temperature profiles for Gl 581c. Dash and dotted-line: dry atmosphere with $P_{\mathrm{s}}=1$ bar and 50 ppmv of $\mathrm{CO}_{2}$, Dashedline: moist atmosphere with $P_{\mathrm{s}}=1$ bar, $50 \mathrm{ppmv}$ of $\mathrm{CO}_{2}$, and same water-vapor mixing ratio as in the Earth atmosphere, and solid line: moist atmosphere with $P_{\mathrm{s}}=1$ bar, $50 \mathrm{ppmv} \mathrm{CO}_{2}$, fixed relative humidity same as in Manabe and Wetherald (1967). A cloud layer is located between 700 and $100 \mathrm{hPa}$ marked by horizontal dotted-lines. b) Vertical profiles of water-vapor volume mixing ratio. The solid and dashed-lines in $\mathbf{b}$ ) correspond to the solid and dashed-lines in a), respectively.
The lack of an effective cold trap for water vapor is also a problem. The dashed and solid lines in Fig. 7 all indicate that the lowest temperatures are higher than $273 \mathrm{~K}$ in the presence of water vapor, suggesting a reduction in the effectiveness of the cold trap for water. It would allow more water vapor into the stratosphere relative to that crossing the tropopause of Earth's atmosphere because there is no dehydration process such as that near Earth's tropopause. As a result, water loss is much faster due to the stronger photolysis of water vapor in the middle atmosphere (Kasting et al. 1993). In addition, Gl 581c may have a much denser atmosphere and higher levels of greenhouse gases since it has a much higher mass than Earth. If it is the case, Gl 581c must have experienced runaway greenhouse, just like Venus.

\section{Discussion and conclusions}

Using a real-gas radiative-convective model, we have studied the $\mathrm{CO}_{2}$ threshold for maintaining the surface temperature of Gl 581d above the freezing point of water. For a dry atmosphere affected by $\mathrm{CO}_{2}$ condensation and $A_{\mathrm{s}}=0.15$, our simulations demonstrate that at least 9.6 bars of $\mathrm{CO}_{2}$ are required. For a moist atmosphere with fixed $\mathrm{RH}$ and $A_{\mathrm{s}}=0.15$, the $\mathrm{CO}_{2}$ threshold is lowered to 6.7 bars because of the greenhouse effect of water vapor. For $A_{\mathrm{s}}=0.30$ and a moist atmosphere with fixed $\mathrm{RH}$, which is close to Earth's planetary albedo, the $\mathrm{CO}_{2}$ threshold is about 11.5 bars. The $\mathrm{CO}_{2}$ threshold in our simulations is slightly higher than that in Wordsworth et al. (2010) and von Paris et al. (2010) because their models all have a stronger greenhouse effect for a moist atmosphere. Our comparison indicates that one of the major factors in causing the differences in $T_{\mathrm{s}}$ is presumably the new parameterizations for $\mathrm{CO}_{2}$ and water vapor absorption in their models. We have also found that collisioninduced $\mathrm{CO}_{2}$ absorption in Wordsworth et al. (2010) has slightly weaker greenhouse effect.

In our simulations for Gl 581d, we have not considered the radiative effect of clouds of both $\mathrm{CO}_{2}$ and water, which also play important roles in constraining $T_{\mathrm{S}}$. Liquid water clouds reflect incident shortwave radiation and cool the surface, whereas ice clouds of $\mathrm{CO}_{2}$ and water have greenhouse effects and warm the surface. In particular, $\mathrm{CO}_{2}$ ice clouds in a high- $\mathrm{CO}_{2}$ atmosphere have a strong greenhouse effect on the surface. As shown by Forget and Pierrehumbert (1997), $\mathrm{CO}_{2}$ ice clouds were a possible factor in causing the greenhouse effect and warming early Mars' surface. We do not study the influence of clouds on the $T_{\mathrm{s}}$ of Gl 581d because the way in which clouds vary depending on $\mathrm{CO}_{2}$ levels is not known for the one-dimensional model (Kasting \& Ackerman 1986).

It is not unrealistic for Gl 581d to contain $\mathrm{CO}_{2}$ at around 10 bars in its atmosphere because it has a minimum mass of about 10 times greater than that of Venus, which has up to 90 bars of $\mathrm{CO}_{2}$ in its atmosphere. However, the maintenance of the high level of $\mathrm{CO}_{2}$ in the atmosphere of Venus is due to the lack of liquid water, which allows $\mathrm{CO}_{2}$, produced in turn by volcano eruptions, to become accumulated in the atmosphere. As long as there is permanent liquid water on the surface of a planet, the water cycle becomes active, removing $\mathrm{CO}_{2}$ from the atmosphere by means of weathering reactions and leading to a low level of $\mathrm{CO}_{2}$ (Walker et al. 1981; Kasting \& Ackerman 1986; Kasting 1988). For example, the formation of the Snowball Earth in the Neoproterozoic era was proposed to be caused when $\mathrm{CO}_{2}$ dropped to about 100 ppmv in Earth's atmosphere because of active weathering reactions (Hoffman \& Schrag 2000; Pierrehumbert 2004). The question then arises of how $\mathrm{CO}_{2}$ can be maintained at a level around 10 bars for Gl 581d in the presence of liquid water. One possibility is that the surface of Gl 581d is mostly covered by ocean, in which case the carbonate-silicate cycle could be inefficient, as suggested by Selsis et al. (2007). It is likely that Gl 581d is mostly covered by ocean because as the mass of the planet increases so does the water-to-surface ratio (Lissauer 1999). Given that Gl 581d resembles an aqua-planet, its surface albedo would be correspondingly lower, and the required minimum $\mathrm{CO}_{2}$ level would also be lower than the above results. It is also possible that the climate system of Gl 581d oscillates between the snowball and habitable regimes driven by the carbonate-silicate cycle.

We have found that our simulations imply that Gl 581c is too hot to be habitable. Since its radiative equilibrium temperature is already very high, even a weak greenhouse effect can readily increase its surface temperature above the threshold of runaway greenhouse. The high planetary albedo caused by extremely thick clouds seems unrealistic. The lack of an effective 
cold trap for water would lead to rapid loss of water. All of these results suggest that Gl 581c had very likely undergone runaway greenhouse like Venus. We note that all our simulations have been performed without considering either the eccentricity or the obliquity. The results here also do not reject the possibility of habitable areas in the transition regions between day and night hemispheres, where the temperature is neither too high nor too low, given that Gl 581c is a synchronously rotating planet. More detailed and qualitative discussion about these aspects can be found in Selsis et al. (2007).

The model predictions discussed in this paper are testable by observations. Given that Gl 581c is a Venus-like planet because it has experienced runaway greenhouse, it should display the distinctive characteristics of Venus, such as a high albedo with blue and ultraviolet broadband absorption, $\mathrm{SO}_{2}$ absorption band due to $\mathrm{H}_{2} \mathrm{SO}_{4}$ clouds, and the absence of $\mathrm{H}_{2} \mathrm{O}$ absorption bands, which are detectable (Mills et al. 2007). The presence or absence of $\mathrm{H}_{2} \mathrm{O}$ and $\mathrm{CO}_{2}$ on Gliese 581c and $\mathrm{Gl} 581 \mathrm{~d}$ may be established by observations such as those for the hot Jupiter HD 189733b (Tinetti et al. 2007; Swain et al. 2009).

Acknowledgements. We are grateful to Prof. Douglas Lin who made helpful comments on an early version of the paper. We thank the anonymous reviewer for careful reviews and very helpful comments, which lead to important corrections and improvements of the paper. This work is supported by the National Basic Research Program of China (973 Program, 2010CB428606), the National Natural Science Foundation of China (40875042, 41025018), and the Ministry of Education of China (20070001002).

\section{References}

Baranov, Y. I., Lafferty, W. J., \& Fraser, G. T. 2004, J. Mol. Spectrosc., 228, 432 Forget, F., \& Pierrehumbert, R. T. 1997, Science, 278, 1273
Goody, R. M., \& Yung, Y. L. 1989, Atmospheric Radiation: Theoretical Basis. 2nd Ed. (Oxford University Press)

Gruszka, M. \& Borysow, A. 1998, Molecul. Phys., 93, 1007

Hoffman, P. F., \& Schrag, D. P. 2000, Sci. Am., 282, 62

Ingersoll, P. 1969, J. Atmos. Sci., 26, 1191

Kasting, J. F. 1988, Icarus, 74, 472

Kasting, J. F., \& Ackerman, T. P. 1986, Science, 234, 1383

Kasting, J. F., Pollack, J. B., \& Ackerman, T. P. 1984a, Icarus, 57, 335

Kasting, J. F., Pollack, J. B., \& Crisp, D. 1984b, J. Atmos. Chem., 1, 403

Kasting, J. F., Whitmire, D. P., \& Reynolds, R. T. 1993, Icarus, 101, 108

Li, K.-F., Pahlevan, K., Kirschvink, J. L., \& Yung, Y. L. 2009, Proc. Nat. Acad. Sci., USA, 106, 9576

Lissauer, J. J. 1999, Nature, 402, C11

Manabe, S., \& Strickler, R. F. 1964, J. Atmos. Sci., 21, 361

Manabe, S., \& Wetherald, R. T. 1967, J. Atmos. Sci., 24, 241

Mayor, M., Bonfils, X., Forveille, T., et al. 2009, A\&A, 507, 487

McKay, C. P., Pollack, J. B., \& Courtin, R. 1991, Science, 253, 1118

Mills, F. P., Esposito, L. W., \& Yung, Y. L. 2007, in Exploring Venus as a Terrestrial Planet, ed. L. W., Esposito, E., Stofan, T., Cravens, American Geophysical Union, Washington, DC, 73

Mischna, M. A., Pavlov, A. A., \& Kasting, J. F. 2000, Icarus, 145, 546

Pavlov, A. A., Kasting, J. F., Brown, L. L., Rages, K. A., \& Freedman, R. 2000, J. Geophys. Res., 105, 11981

Pierrehumbert, R. T. 2004, Nature, 429, 646

Ricchiazzi, P., Yang, S., Gautier, C., \& Sowle, D. 1998, 79, 2101

Selsis, F., Kasting, J. F., Levrard, B., et al. 2007, A\&A, 476, 1373

Swain, M. R., Vasisht, G., Tinetti, G., et al. 2009, Astrophys. J., 690, L114

Tinetti, G., Vidal-Madjar, A., Liang, M. C., et al. 2007, Nature, 448, 169

Toon, O. B., McKay, C. P., Ackerman, T. P., \& Santhanam, K. 1989, J. Geophys. Res., 94, 16287-16301

Udry, S., Bonfils, X., Delfosse, X., et al. 2007, A\&A, 469, L43

US standard atmosphere, 1976, US Government printing office, Washington DC Walker, J. C. G., Hays, P. B., \& Kasting, J. F. 1981, J. Geophys. Res., 86, 9776

von Bloh, W., Bounama, C., Cuntz, M., \& Franck, S. 2007, A\&A, 476, 1365

von Paris, P., Gebauer, S., Godolt, M., et al. 2010, accepted by A\&A, 522, A23

Wordsworth, R. D., Forget, F., Selsis, F., et al. 2010, A\&A, in press

Yung, Y. L., \& DeMore, W. D. 1999, Photochemistry of Planetary Atmospheres (Oxford University Press) 